\section{Late Palaeozoic Wrangellian palaeomagnetic directions north or south seeking?}

IN a recent Nature article Panuska and Stone ${ }^{1}$ report that they have resolved the polarity ambiguity in the late Palaeozoic palaeomagnetic data for Wrangellia. They have measured magnetic directions from the Lower Permian Hasen Creek Formation and the Lower Permian-Pennsylvanian Station Creek Formation which they conclude would place Wrangellia north of the Equator in the late Palaeozoic. They reason that since the Earth's field was predominately reversed at this time, any directions measured from these rocks would point to the reversed polarity geomagnetic pole, that is, the geographic south pole. However, their interpretation may be at odds with their data. Most palaeomagnetic studies report palaeomagnetic data just as it was measured from the rocks. If the southwest and down directions (Table 1 in ref. 1) are what was measured in the late Palaeozoic Wrangellia rocks, then these reversed directions would suggest that Wrangellia was south of the Equator and not north of the Equator, as Panuska and Stone $^{1}$ conclude. Two statements in Panuska and Stone's ${ }^{1}$ article suggest that the southwest and down directions reported in Table 1 are not those that were actually measured. Both occur on page 562. "Thus taking into account the reversed geomagnetic polarity, the VGPs corresponding to the mean vectors plotted in Fig. 2 represent an estimate of the location of the north geographical pole for that time." and "The polarity of the tabulated data represent the equivalent of today's Northern Hemisphere pole."1 Although these statements may imply that the data have been changed to correspond to the authors' polarity interpretation, nowhere do the authors clearly state what directions were actually measured.

The reason for this comment is to point out that it is misleading and confusing if the data are not reported exactly as measured. Interpretations should be made only after the reader has access to the raw data. In this particular case it is important to know if the southwest and down directions were actually measured in the rocks, in which case Panuska and Stone's ${ }^{1}$ interpretation is incorrect, or if the opposite polarity were measured.

\section{K. P. KODAMA}

Department of Geological Sciences, Lehigh University,

Bethlehem, Pennsylvania 18015, USA

1. Panuska, B. \& Stone, D. B. Nature 293, 561-563 (1981)
PANUSKA AND STONE REPLY-We welcome the opportunity to clear up the misunderstanding of data that we reported in our 1981 paper. The directions of the north-seeking magnetic vectors that we measured in the Skolai Creek rocks àre actually northeasterly and negative (up). Since only summary data (that is, mean directions) were reported in our Table 1, and not the raw data, we inverted the mean directions in the interest of saving space by avoiding the use of separate tables for measured directions and inferred directions and "avoiding confusion" by reporting the probable polarity. The two statements cited by Kodama were intended to indicate that the measured directions were inverted for this paper because of the predominant reversed geomagnetic polarity during deposition of the Skolai Creek rocks. We have apparently failed in our attempt to avoid confusion and we thank Dr Kodama for bringing this to our attention.

To summarize, we measured northeast and negative (up) magnetic directions in the Skolai Creek rocks. These directions were inverted to the southwest and positive (down) directions reported in Table 1 because of the reversed geomagnetic polarity during the Permo-Carboniferous. Thus, we stand by our original interpretation of a Northern Hemisphere location for Wrangellia in late Palaeozoic time.

\section{BRUCE C. PANUSKa} DAVID B. STONE

Geophysical Institute

University of Alaska,

Fairbanks, Alaska 99701, USA

\section{Noise and recognizability of coarse quantized images}

WE are pleased that Morrone et al. ${ }^{1}$ found it still worthwhile a decade later to elaborate on an experiment by Harmon and $\mathrm{Julesz}^{2}$, even though they disagree with our conclusions. We comment only because: (1) from Morrone et al. the reader might assume that Harmon and Julesz had critical-band masking as their only explanation ${ }^{2} ;(2)$ they failed, so far, to disprove critical-band masking; and (3) their demonstration does not seem convincing. We comment now in detail along those lines.

(1) Harmon and Julesz $z^{2}$ offered two possible explanations for the inability to see Abraham Lincoln's face in the blockquantized image. One was discussed by us at length, but not quoted by Morrone et $a l^{1}$, although they give a similar explanation. We assumed that the visual system was very sensitive to line contours at the boundaries of the quantized blocks and that these contours masked the lowspatial-frequency image. Our alternative explanation was critical-band masking, since we showed that the removal of an annulus-shaped spectrum from the Fourier spectrum of the block-quantized image restored the visibility of Lincoln's face. (This annulus had an inner radius $f_{0}$ and outer radius $2 f_{0}$, where $f_{0}$ is the highest frequency in the Lincoln image.) Thus the quantization noise of the middlefrequency annulus next to the image spectrum was adequate to mask the image. This restoration of recognition was the more remarkable since some of the line contours around the quantized blocks were still visible. We also demonstrated that by filtering out the high frequencies outside the disk with $2 f_{0}$ radius, the image of Lincoln remained hidden. This filtering removed the sharp contours at the boundaries of the quantized blocks, yet the face remained masked. It was these findings that persuaded us to consider critical band masking as an alternative to the trivial first explanation.

(2) Critical-band masking occurs for one-dimensional gratings and noise as shown by Stromeyer and Julesz ${ }^{3}$. This was recently reconfirmed by Wilson et al. ${ }^{4}$. In another study, Phillips and Wilson showed that for 2.5-6 cycles per deg test and masking gratings, the orientational tuning curves (half-amplitude bandwidths) are as shallow as 25 arc deg. While Morrone et al. did not specify the viewing distances in their experiment, one can assume that much of their 'windmill' noise falls in the above mentioned spatialfrequency range. It is not surprising that the windmill noise only $22.5^{\circ}$ away from the axes of the two-dimensional Fourier domain will mask the masking noise; this is where the quantization-noise spectra are concentrated. Such a masking of the masker (disinhibition) was reported by Stromeyer and Julesz ${ }^{3}$.

According to our comment, we would like to see a demonstration by Morrone et al. with windmill noise restricted to the diagonals (say, between 40 and 50 arc deg.). Only if this noise were to unmask the original image would we regard the idea of critical-band masking as being challenged. Even then many questions would still be left unsettled. For instance, we used concentric filters with circular symmetry that yielded the same 1-octavewide critical band for two-dimensional stimuli that were found by Blakemore and Campbell $^{6}$ for one-dimensional stimuli. Whether asymmetric filtering can be used without causing some nonlinear effects remains to be seen. The finding by Daug$\operatorname{man}^{7}$ and Phillips and Wilson ${ }^{5}$ that twodimensional spatial frequency channels are inseparable in their polar coordinates should caution us. 